\title{
Joint Modeling of Mobility and Communication in a V2V Network for Congestion Amelioration
}

\author{
Pritesh Patwa \\ Microsoft Corporation \\ Redmond, Washington, USA \\ ppatwa@microsoft.com
}

\author{
Rudra Dutta \\ Computer Science, North Carolina State University \\ Raleigh, NC, USA \\ dutta@csc.ncsu.edu
}

\begin{abstract}
Vehicular networks are emerging as an exciting new area of pervasive networking. Roadway congestion amelioration is one of the attractive applications of such a network. The mobility of vehicles on the roadway has been modeled in literature, but the effect of the communication network on the mobility patterns has not been addressed so far. We present such a model of the mobility that integrates the effects of the network. Our model is based on an earlier model using a Enskog dense gas modeling of the traffic flow. Our solutions show that the model is realistic and numerical simulations also verify the results. We also note that this application can cause oscillation waves in the traffic flow, which have so far not been recognized in literature.
\end{abstract}

\section{INTRODUCTION}

Vehicular networking is a swiftly emerging pervasive computing research and application areas. These networks have also been called Vehicular Ad-hoc Networks (VANETs) or CarHoc (for Ad-hoc Car networks). Automotive communication can be classified in general as vehicle-to-vehicle (V2V) or vehicleto-infrastructure (V2I). There have already been efforts in deployment of specific implementations [1]-[5] as well as in standardization [6], [7]. Obtaining a good model of the mobility of vehicles is obviously an essential prerequisite to many other studies of V2V and V2I networks. There has been significant work in modeling the mobility of cars along highways, or other road networks [8]-[12]. However, these have all been in the context of traditional road traffic, and there has been no study of how ubiquitous applications may change such flows. Similarly, networking architectures for ubiquitous applications have been proposed and studied; e.g. congestion detection algorithms has been described in literature [13]-[16], and network and MAC layer approaches suitable for use in this context [17], [18] have also been described; however, again the efficiency of these approaches have only been analyzed by making the simplifying assumption that they do not change the mobility pattern. There has been as yet no attempt at modeling the mobility pattern of vehicles taking the effect of the ubiquitous application into account.

In this paper, we attempt such a joint modeling. It is worth considering whether such a study is useful; i.e., does the ubiquitous application sensibly change the mobility model? After all, the mobility of the cars is determined by their drivers, on timescales of seconds, while the ubiquitous network connects the computers embedded in the cars, and operates on timescales of milliseconds. That the network could potentially affect the mobility pattern of the cars is not intuitively obvious, and this is in part what we investigate in this paper.

As a representative intelligent transportation application, consider the roadway congestion detection and notification application mentioned above. Radio-enabled automobiles communicate with each other to exchange location information and detect when the average velocity of traffic at some point on the highway falls below a certain threshold, which is considered the onset of congestion. A congestion notification is then transmitted car-to-car, so that vehicles approaching the congestion but not yet inside the congested region can display warnings to their drivers, who might choose to exit the highway and take an alternate route. This is referred to as amelioration of congestion, because while the roadway congestion cannot be eliminated altogether, the number of cars which suffer from this congestion is minimized, and the congestion is resolved sooner, minimizing wastage in driver time and fuel.

But by exiting, the vehicles change their mobility pattern; thus the ubiquitous application exerts an influence on the spatial distribution of vehicles. We already know that connectivity and other network characteristics can depend on the spatial distribution. Thus there is a feedback loop between the mobility of the cars and the radio communication, which has thus far been neglected in literature. To know whether such feedback can have any deleterious effect, it is necessary to model the mobility of the cars under the congestion amelioration application.

Note that previous models of mobility does not provide an answer to this question, because they do not jointly model the effect of communication on the driver's decisions. Further, our model is pertinent in the short timescale, which is the timescale of both the roadway congestion phenomena and network communication. Driver decisions are also affected by human-scale phenomena such as TV and broadcast radio announcements, phone calls between friends, etc.; these operate at timescales much larger than mobility.

The rest of this paper is organized as follows. Next, we briefly review some pertinent literature. In Section III, we describe the essentials of the mobility model presented in [10], on which our joint model is based. Section IV presents the evolution of our joint model. Solutions of the model under various scenarios are presented in Section V, where we show the effect of the feedback loop on the mobility pattern, which has not been recognized before and may be undesirable. As 
verification of the qualitative nature of our model, we created a simulation of the system at the microscopic level, some results of the simulation are also presented. Section VI concludes the paper.

\section{Context And Prior Work}

Unlike traditional networks for similar use, such as cellular wireless communication between vehicles and a monitoring center [4] [5], VANETs are decentralized and do not require a large pre-installed infrastructure to benefit users. For this reason VANETs are expected to be among the earlier deployed solutions in Intelligent Transportation Systems (ITS). VANETs are based on short-range wireless communication between vehicles, e.g., Dedicated Short Range Communication (DSRC) [3] and IEEE 802.11 [6]. Other technologies at the MAC layer include Bluetooth and cellular networks.

At the network layer, routing and forwarding in VANETs is unique due to high mobility of nodes, which results in frequently changing network topology. Several routing protocols [17]-[20] have specifically been proposed for V2V communication, such as Role Based Multicast (RBM) [19] and Inter-Vehicle Geocast [20].

Traffic congestion is said to occur when vehicle speeds are much reduced, drive times are significantly increased, and fuel burned unnecessarily. There are several incident detection algorithms that are being currently deployed on the freeways. A comprehensive list can be found in [14]. Many depend on a dynamic group membership, such as [15], [16]. Congestion is detected when the speed of any reporting Communication Enabled Car (CEC) falls below a certain threshold.

As we remarked above, modeling traffic flow (without any reference to VANET applications) has received attetion in literature. Traffic models available in literature can be classified [21] according to the following fundamental characteristics: 1. Scale of independent variables: A model can define quantities which are continuous, discrete or semi-discrete. 2. Level of detail: Microscropic models involve quantities that represent individual cars and their motion, whereas macroscopic models consider quantities that are properties of a large collection, such as average velocity, or emergent quantities like the pressure of the flow at some point of the roadway. Intermediate levels of representation also exist. 3. Representation of the processes: Models can be deterministic or stochastic. The former models have no random variables implying that all quantities in the model are defined by deterministic relationships. Stochastic models incorporate processes that include random variables. 4. Analytical or Simulation: Models can be either analytical solutions of sets of equations, or they can be simulation based. 5. Area of application: The model may describe the dynamics of its entities for a single lane or a multi-lane freeway, a downtown street, an entire traffic network, etc.

A continuous microscopic single-lane car-following model, named Intelligent Driver Model (IDM), has been simulated in [8], using empirical boundary conditions which are defined essentially by a function that specifies how much the acceleration (positive of negative) of a vehicle will be under different variables such as distance from next leading car, approach rate, etc.. Kesting et al. [9] have proposed a general scheme of lanechanging rules for a wide class of microscopic models using a similar approach. An essential additional parameter in this lanechanging model is the "politeness factor" $p$, which represents the degree of cooperation when considering lane changes. The model in [10] is based on reaction thresholds which are dependent on the velocities of cars. The cars change velocity and lanes instantaneously once certain reaction thresholds are crossed. Both [11] and [10] have derived models at an intermediate level of detail from the corresponding microscopic models by modeling the interaction among vehicles as a statistical phenomenon as that among gas molecules.

At a macroscopic level, traffic is described at a high level of aggregation as a fluid flow, without distinguishing its constituent parts. Flow, density, and velocity are considered as fundamental quantities. Individual vehicles and their motions, such as lane change, are not explicitly represented. Helbing [12] has presented a single-lane macroscopic model derived by application of equations similar to Navier-Stokes equations. The model consists of a set of partial differential equations which describe the change in velocity, flow, etc. at any point on the roadway at any moment in time. Any consistent solution to the system of equations represents a history of the flow over a region and period of interest. Initial and/or boundary conditions can be imposed to obtain a particular solution of interest. The macroscopic model of [10] is similar, except Enskog equations, a form of Navier-Stokes equations suitable for dense gases, are used. This is claimed to be a more realistic model for traffic flow, because such gases can attain equilibria under inhomogeneous conditions (as opposed to thinner gases which can only attain homogeneous equilibrium).

\section{KLAR's Model of MobiLITy}

In this secction, we briefly describe the model advanced in [10], and further investigated in [22], which we adopt as the basis of our joint modeling. For details, we refer the reader to the original work.

The model is built in the context of a single stretch of multilane highway. No Exits are considered in this modeling, cars enter only at the tail end of the stretch of highway which is considered the region of interest, and leave only at the head end. These ends are arbitrary and merely demarcate the region of interest; the actual highway is expected to extend beyond either end.

The microscopic part of the model is based on an interpretation of driver behavior as detailed in Algorithm 1. Effectively, the driver decisions are characterized by various thresholds; if the distance to the next car in front of a given vehicle $C$ is beyond a certain threshold, the driver of $C$ does not perceive him/herself as being constrained by the car in front. At successively close thresholds, $C$ attempts to switch to the left (faster) lane, the car in front of $C$ attempts to switch to the right lane, and $C$ is forced to start braking. Under unconstrained conditions, the car $C$ will accelerate to its desired velocity according to the following rules: at every instant, the driver 
chooses a random fraction $\xi$ between 0 and 1, and increases its velocity by $\xi\left(v_{d}-u\right)$, where $v_{d}$ is its desired velocity, and $u$ is its current velocity. The desired velocities of individual cars is assumed to be distributed according to some reasonable distribution.

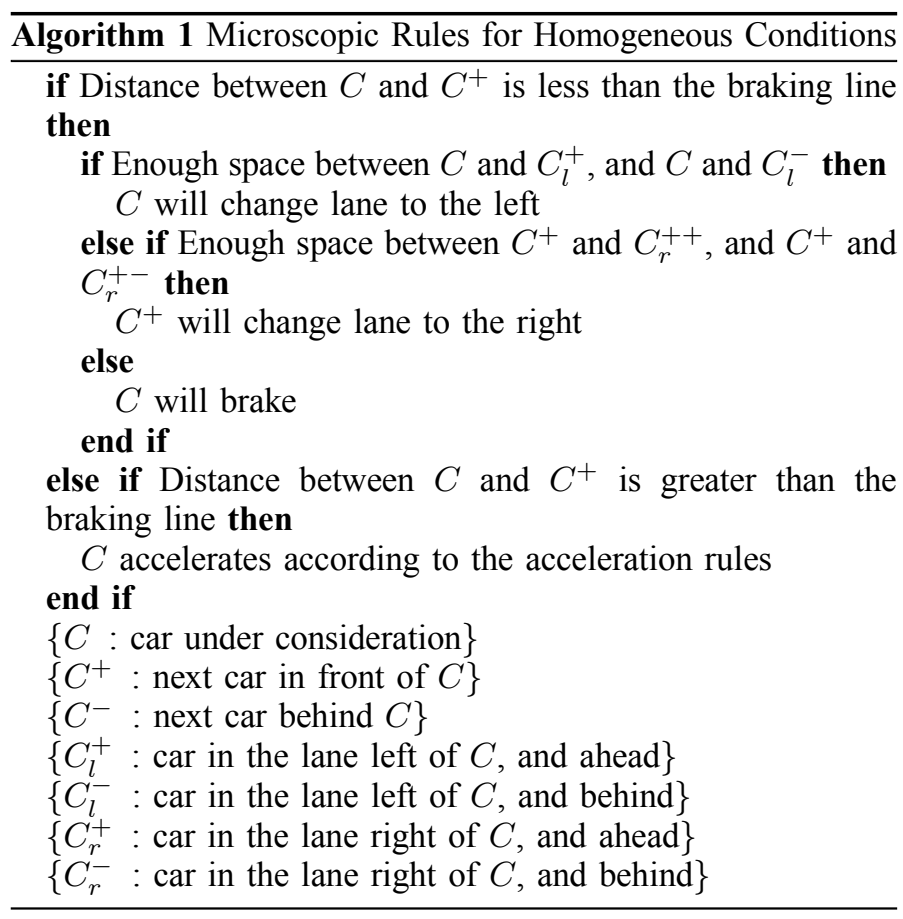

This micromodel is utilized to develop a kinetic model (intermediate level) according to Enskog gas theory. This part of the model yields equilibirium equations that effectively provide feasible value pairs for different aggregate quantities characterizing the traffic. The fundamental aggregate quantities that are of interest are:

- $\rho$ The density of traffic,

- $u$ The velocity of traffic.

Both of the above are functions of the position along the highway, and time; and are also defined separately for each lane of the highway. These are indicated by functional arguments $x$ and $t$ and subscript $\alpha$ respectively, where required. Another fundamental quantity is the flow of the traffic $m$; however, this is not an independent quantity and is defined as $m=\rho u$. The equilibrium conditions are expressed by relations between these quantities with the superscript $e$; for example, $u^{e}(\rho)$ indicates the mean equilibrium velocity corresponding to a density of $\rho$. As may be expected, the equilibrium velocity decreases with increasing density, slowly at first, then precipitately. We recall that with the Enskog modeling, it is possible for different places on the highway to inhabit different points on the equilibrium curve; that is the equilibrium may be inhomogeneous. Also, the rate of lane changes from each lane to each neighboring lane is quantified into constants from stochastic considerations.

Next, these equilibrium conditions are utilized to obtain a macromodel which can predict the values of the aggregate quantities over the period and region of interest. The model is derived as a set of partial differential equations. For our purposes, it is necessary only to focus on the final model, which is given by (1) and (2). The significance of the various parameters are as follows:

$\frac{1}{T^{L}}$ : Rate of lane change to the left, from lane $\alpha$ to $\alpha+1$

$\frac{1}{T_{\alpha}^{R}}$ : Rate of lane change to the right, from lane $\alpha$ to $\alpha-1$

$\delta_{i, j}^{\alpha}$ : The Kronecker delta that enforces the asymmetry at the boundary lanes; no lane change to the left is possible on the left-most lane and no lane change to the right is possible on the right-most lane.

$$
\delta_{i, j}= \begin{cases}1 & \text { if } i=j \\ 0 & \text { otherwise }\end{cases}
$$

$\frac{1}{T^{e}\left(\rho_{\alpha}\right)}$ : Sum of braking and acceleration interaction frequencies between vehicles at equilibrium

$u^{e}\left(\rho_{\alpha}\right)$ : Mean velocity at equilibrium

$p^{e}\left(\rho_{\alpha}\right)$ : Equilibrium pressure

$A^{e}\left(\rho_{\alpha}\right)$ : Integrated Enskog coefficient at equilibrium

The Density Equation, (1), predicts the rate of change of density $\rho_{\alpha}$, in lane $\alpha$, and is influenced by the densities in the immediate surroundings on the same lane and the adjacent lanes. Terms on the right-hand side of the equations, define the sources and sinks due to lane changing, e.g., $\frac{1}{T_{\alpha-1}^{L}} \rho_{\alpha-1}$ is the gain in the density on lane $\alpha$, due to left lane change from lane $\alpha-1$ and $\frac{1}{T_{\alpha}^{R}} \rho_{\alpha}$ is the loss in the density on lane $\alpha$, due to right lane change from lane $\alpha$. Similarly, the $\partial_{x}\left(\rho_{\alpha} u_{\alpha}\right)$ term on left hand side of the equation defines the effect due to the densities at immediate surroundings on the same lane.

The Flow Equation (2) can be interpreted in a similar way. It models the rate of change of flow $\left(\rho_{\alpha} u_{\alpha}\right)$, and is influenced by the flows in the immediate surroundings on the same lane and the adjacent lanes. In the model, it is assumed that the lane changes occur only due to a desire to avoid braking, and therefore, the lane changing rates $\left(\frac{1}{T_{\alpha}^{L}}\right.$ and $\left.\frac{1}{T_{\alpha}^{R}}\right)$ are dependent on the probability of a lane change and the braking interaction frequencies between the vehicles. While deriving macroscopic fluid dynamic equations, authors have defined closure relations for the gas-kinetic equations using the stationary (equilibrium) solutions. Due to such an approach, equilibrium parameters like $u^{e}\left(\rho_{\alpha}\right), A^{e}\left(\rho_{\alpha}\right), p^{e}\left(\rho_{\alpha}\right)$ and $\frac{1}{T^{e}\left(\rho_{\alpha}\right)}$ influence the dynamics of fundamental quantities even in inhomogeneous conditions. That is, the assumption of quasi-static equilibrium, standard in gas dynamics, has been made; and the Enskog modeling lends confidence that this assumption remains a good one even though the condition of the flow can clearly become inhomogeneous.

In [22], the authors have investigated the behavior of this model under two broad conditions. In the first case, all lanes remain open over the entire period of interest, and initially the road is empty. Cars enter from the tail end of the region of interest at a constant average flow and velocity. Over time, the density of the flow is seen to rise over the entire stretch of highway, as expected. In the second case, the third (leftmost) lane is closed. Traffic approaching the obstruction if forced to switch to the second lane, increasing density in that lane as 


$$
\begin{gathered}
\partial_{t}\left(\rho_{\alpha}\right)+\partial_{x}\left(\rho_{\alpha} u_{\alpha}\right)=\left(\frac{1}{T_{\alpha-1}^{L}} \rho_{\alpha-1}-\frac{1}{T_{\alpha}^{R}} \rho_{\alpha}\right)\left(1-\delta_{\alpha, 1}\right)+\left(\frac{1}{T_{\alpha+1}^{R}} \rho_{\alpha+1}-\frac{1}{T_{\alpha}^{L}} \rho_{\alpha}\right)\left(1-\delta_{\alpha, N}\right) \\
\partial_{t}\left(\rho_{\alpha} u_{\alpha}\right)+\partial_{x}\left(p^{e}\left(\rho_{\alpha}\right)+\rho_{\alpha} u_{\alpha}^{2}\right)+\partial_{x}\left(A^{e}\left(\rho_{\alpha}\right)\right)= \\
\left(\frac{1}{T_{\alpha-1}^{L}} \rho_{\alpha-1} u_{\alpha-1}-\frac{1}{T_{\alpha}^{R}} \rho_{\alpha} u_{\alpha}\right)\left(1-\delta_{\alpha, 1}\right)+\left(\frac{1}{T_{\alpha+1}^{R}} \rho_{\alpha+1} u_{\alpha+1}-\frac{1}{T_{\alpha}^{L}} \rho_{\alpha} u_{\alpha}\right)\left(1-\delta_{\alpha, N}\right)+\frac{1}{T^{e}\left(\rho_{\alpha}\right)} \rho_{\alpha}\left[u^{e}\left(\rho_{\alpha}\right)-u_{\alpha}\right]
\end{gathered}
$$

well as behind. Over time, this effect also percolates to the first lane. The interesting outcome is that the model correctly predicts that this creates the onset of congestion. In the model, as in reality, the average velocity of the vehicles in the vicinity of the lane closure falls drastically, and the flow through that region of the highway falls. If the lane remains closed, the congested zone (the region over which the average velocity is low) grows backward along the road. This behavior is taken as validation of the qualitative behavior of the model. The authors of [10], [22] have not included a numerical simulation of the underlying micromodel as verification.

\section{JOINT MODELING}

\section{A. Assumptions}

We now present our enhancement of this model to represent the feedback effect with the communication system. At the outset, we recognize that there are several issues that need to be considered in this connection:

1) Fraction of CECs: During the initial deployment of telematics in the market, not all the vehicles will be equipped with radios and GPS. Hence, one can expect sparsely distributed nodes on the road. Therefore, in the joint model we need to account for a variable fraction of CECs.

2) Efficiency of the protocols on Network, MAC and Physical layers: The efficiency of communication protocols in VANET environment will significantly influence the extent up to which telematics are able to improve the driving conditions. As we mentioned above, there has been significant research in this context, and efficient strategies are likely to be available.

3) Efficiency of the Incident detection and communication algorithms: Incidents like congestion, bad weather, accidents etc., can be detected via driver assistance, or automatically by equipping the vehicles with appropriate capabilities. The research community [23] has widely addressed on Automatic Incident Detection (AID) algorithms, and it may be safely assumed that incident detection will be very efficient.

4) Driver behavior in response to the warning messages: The manner in which drivers respond to the warning messages depends on the severity of the incident and its impact on the safety and travel times of the driver. Hence, the driver decisions will affect the extent up to which communication affects the mobility of vehicles. Since there is insufficient history regarding this, our model should accommodate various possible extent of driver response.
Motivated by the above considerations, we make the assumptions listed below, classified into two broad categories, depending on whether they are related to the communication networking or to the traffic flow.

\section{Communication Network Based Assumptions}

With regard to communication networking, we make following assumptions:

1) All the CECs are equipped with similar radios (having omni-directional antenna) and GPS, and therefore have similar communication ranges and consistent position coordinates. Also, CECs always know their current positions and can detect other CECs within communication range in negligible time.

2) Efficient network layer protocols and the network access (MAC and physical layer) protocols are already deployed. At the network layer, geographical positions and directions of motion of the vehicles are used for addressing the vehicles, and the routing algorithm is similar to IVG. For the purpose of focusing on the mobility, we assume that addressing and routing are flawless. Communication delays are in the range of milliseconds and therefore can be neglected.

3) All the CECs have consistent communication and congestion detection algorithms installed on them, hence, they can always correctly interpret the messages that are forwarded to them.

4) CECs are always able to communicate with other CECs within their communication range.

\section{Traffic State Based Assumptions}

With regard to the traffic flow, traffic patterns, and traffic states, we make following assumptions:

1) Traffic congestions of continuous form are considered only. In continuous form, only a single connected region is congested at any time.

2) The inflow of vehicles is uniform on all the lanes. Moreover, at the inlet, CEC density is also uniformly distributed across all the lanes. While strictly unrealistic, this is consistent with previous literature [24], and is known to not significantly impact the realistic nature of the model.

3) No vehicle breakdown or other barrier to traffic flow happens on the freeway.

4) The congestion amelioration application by its very nature forces us to consider the presence of an Exit from the highway, which we incorporate in the model. We assume that after reaching the location of the exit, drivers can exit the freeway instantaneously, irrespective of the lane 
on which they are driving. This is again similar to [24]. We introduce the following additional notation to represent CECs: $\rho^{\mathrm{com}}$ is the density of CECs, and varies with time, place, and lane as well. The fraction of CECs is considered a varying parameter, and is defined only at the tail end of the region of interest; it is denoted by $f^{c o m}$, so that $\rho^{c o m}=\rho f^{c o m}$ at the tail end of the highway. However, $\rho^{\text {com }}$ can obviously vary over the rest of the highway length, and over time. We define $U_{\text {average }}(x)$ to be a continuous function that represents average velocity at any position $x$ on the freeway, and $u_{\text {threshold }}$ defines the minimum velocity threshold, that is, if average velocity at any position on the freeway drops below $u_{\text {threshold, }}$, it is considered congested. At the microscopic level, if the velocity of a CEC drops below $u_{\text {threshold }}$, it detects that it is in traffic congestion.

$L$ represents the length of the unidirectional stretch of the freeway, and $X_{\text {Exit }}$ represents the position of the exit on the freeway. When closure of the leftmost lane is considered, the closure is at a position denoted by $X_{\text {LClose }}$. The direction of motion of the vehicles on the considered freeway section is from the tail end to the head end. In our model, we define two kinds of congestion, represented by actual traffic congestion, Cong $_{a}$, and detected traffic congestion Cong $_{d}$. In this thesis, the term congestion implicitly means actual traffic congestion. The head, Cong head and the tail, Cong $g_{a}^{\text {tail }}$, are defined as the furthest and earliest positions along the freeway that are congested, respectively. Similarly, the head, Cong head and the tail, Cong tail, are defined as the furthest and the earliest positions of the freeway section where the congestion has been detected. Clearly,

$$
\text { Cong }_{a}^{\text {tail }} \leq \text { Cong }_{d}^{\text {tail }} \leq \text { Cong }_{d}^{\text {head }} \leq \text { Cong }_{a}^{\text {head }}
$$

We introduce the concept of Severity of Congestion (SoC) as an objective measure of the subjective perception of drivers, which determines their likelihood of exiting. The SoC is described in detail below The number of CECs between any two positions on an $N$ lane freeway, $x$ and $y, x \geq y$, is represented as, $N u m_{c a r}^{c o m}(x, y, N)$. Obviously, we have

$$
\operatorname{Num}_{\text {car }}^{\text {com }}(x, y, N)=\sum_{\alpha=1}^{N} \int_{y}^{x} \rho_{\alpha}^{\text {com }}(x) d x
$$

$R$ represents the transmission range of the radio on a vehicle.

We assume that information regarding Cong $_{d}$ are propagated in messages that we call the Congestion Warning Message (CWMsg). It essentially consists of the information about length and position of $\mathrm{Cong}_{d}$, and the position on the freeway up to which this message would be relevant and therefore, should be propagated. $\psi$ represents the fraction of drivers that choose to exit the freeway in response to $C W M s g$. Length of the actual and detected congestions are represented by $\triangle C_{o n g}$ and $\triangle$ Cong $_{d}$, respectively, and are the distances between the corresponding heads and tails. Clearly, $\triangle$ Cong $_{d} \leq \triangle$ Cong $_{a}$.

\section{B. Determining Congestion - Actual and Detected}

Whether congestion warning is sent to CECs approaching the lane closure or not will obviously affect the behavior of the drivers, and has to be represented in our model. In turn this is affected by whether congestion has actually occurred or not. To specify the determination of these quantities at the microscopic level, we present the Algorithms 2 and 3. We have implicitly assumed that the spatial distribution of CECs among the complete set of cars (when $f^{\mathrm{com}}$ is less than 1) is uniformly interspersed at the tail end of the region of interest. This is in keeping with the fluid modeling of the traffic in general.

The macroscopic effect of this is as follows. When a CEC detects that its velocity has dropped below $u_{\text {threshold, }}$ and it has not heard CWMsg from any car in front of it, it broadcasts a CWMsg with its own position as head (Cong $\left.g_{d}^{\text {head }}\right)$ and tail $\left(\right.$ Cong $\left._{d}^{\text {tail }}\right)$. If any other CEC in congested area behind this car receives this $C W M s g$, it replaces the Cong tail to its own position and recomputes $\triangle C_{\text {ong }}$, and broadcasts this updated CWMsg. This way, the $C_{o n g}^{\text {tail }}$ is set to the position of the hind most CEC in the Cong $_{a}$, and the Cong head is set to the position of the farthest CEC in Cong $_{a}$ that can be reached by Cong $g_{d}^{\text {tail }}$. Cong $g_{d}^{\text {head }}$ and Cong $_{d}^{\text {tail }}$ define the length of detected congestion $\left(\triangle\right.$ Cong $\left._{d}\right)$.

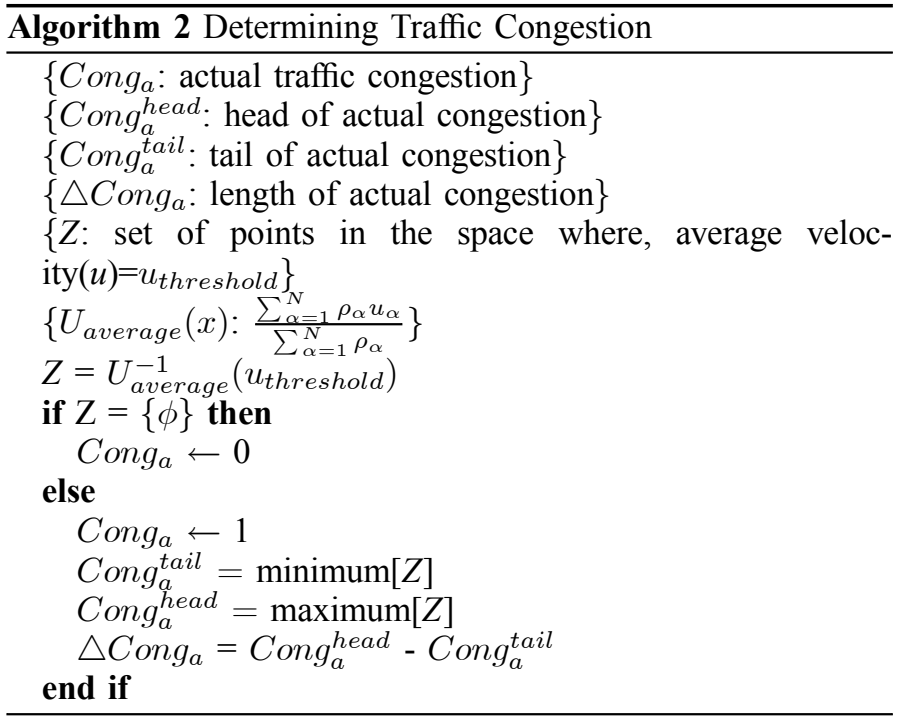

\section{Message Propagation at Macroscopic Level}

The CWMsg is propagated to the target vehicles through communication between CECs that are already inside the congestion and those that lie between the congestion $\left(\mathrm{Cong}_{d}^{\text {tail }}\right)$ and the target vehicles. We create a variable prop to indicate whether message propagation occurs or not. Propagation occurs $($ prop $=1)$ only if these CECs form a connected network. At the microscopic level if the network is not connected (prop $=0$ ), CWMsg can not be propagated to the target CECs. Algorithm 4 shows the determination of propagability at the macroscopic level. We locate the cars in every $R$ length segment between the Cong $_{d}^{\text {tail }}$ and the target vehicles. Moreover, CWMsg is last updated by Cong $_{d}^{\text {tail }}$ and is forwarded till a position up to where it will be relevant. The message is propagated up to Cong $g_{d}^{\text {tail }}-M$ where, $M$ is a constant that represents the extent to which congestion informtion is useful on the actual roadway 


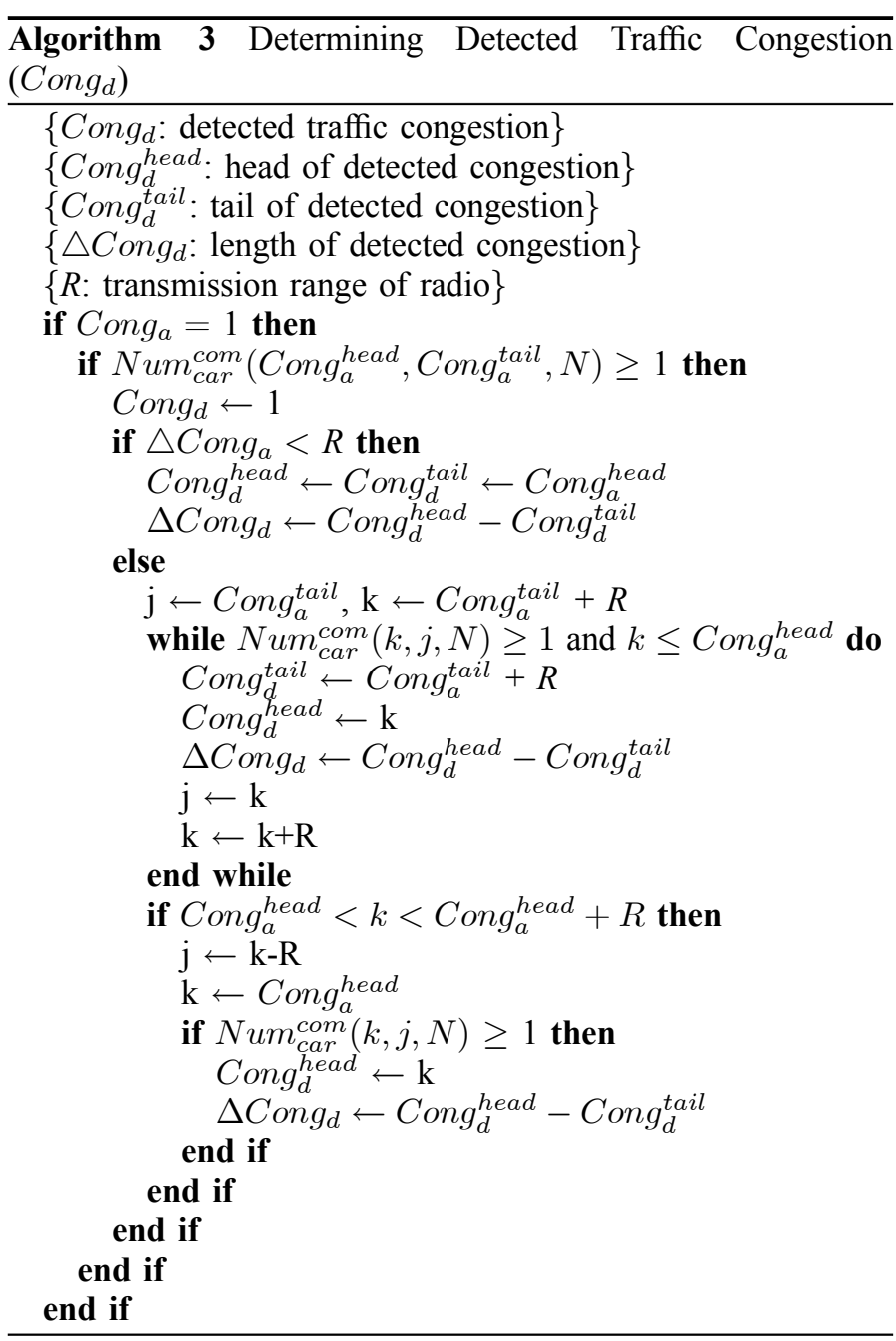

(for example, information may be useful 10 miles from the point of congestion, but is unlikely to be useful 100 miles behind). For the small values of $L$ considered in this paper, $M>L$. If the network is connected, the propagation delay is assumed to be in the range of milliseconds for smaller $L$, and therefore is neglected. We have considered the possibility of congestion extending beyond the $X_{E x i t}$ to be out of scope of the current work.

\section{Modeling Driver Behavior $(\psi)$}

When the target vehicles receive the CWMsg, the drivers may or may not choose to exit the freeway. The fraction of the drivers choosing to exit is influenced by the Severity of Congestion $(\mathrm{SoC})$, which is defined below:

$$
\text { SoC }=q \triangle \text { Cong }_{d} /\left(\text { Cong }_{d}^{\text {tail }}-X_{E x i t}\right)
$$

Here, Cong tail $>X_{\text {Exit }}$ and the parameter $q(>1)$, is a scale factor, that models the greater impact of $\triangle C o n g_{d}$ on the $S o C$, than its closeness to the $X_{\text {Exit }}$.

In turn, $\psi$ represents the driver behavior i.e., the fraction of the cars that choose to exit in response to the $S o C$ and is defined

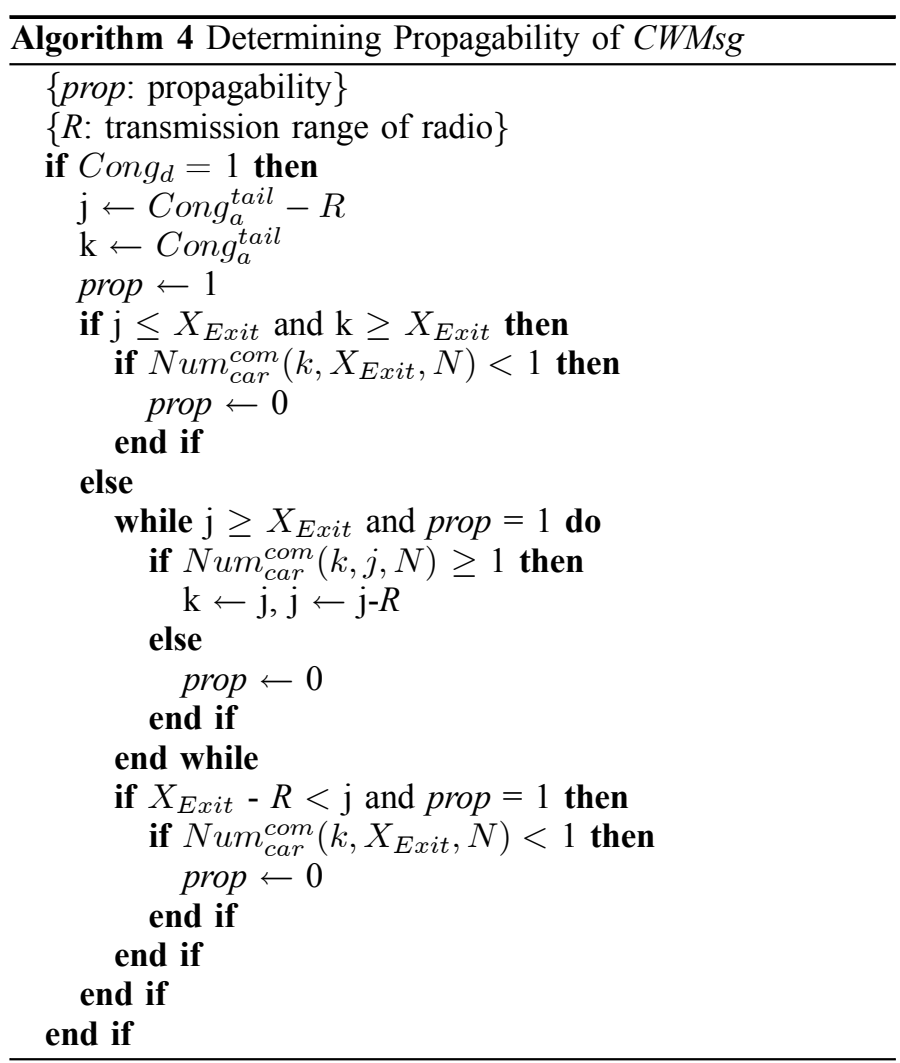

as follows:

$$
\psi=\operatorname{prop}\left[\Phi \frac{1}{1+e^{-a(\operatorname{SoC}-c)}}\right]
$$

where prop is 0 or 1 as defined above. This is a sigmoid function, which correctly represents the non-linearity and saturation of reaction that may be naturally expected, but does not involve discontinuous changes in the function derivative. $\Phi$ represents the maximum fraction of CECs that can exit the freeway at any given time and therefore represents the maximum value that $\psi$ can attain.

At this point, we are ready to specify the joint model. When CECs exit in response to the CWMsg, they affect the dynamics of macroscopic fundamental quantities, $\rho_{\alpha}, \rho_{\alpha}^{c o m}, u_{\alpha}$ and $\rho_{\alpha} u_{\alpha}$. In the new integrated model, this is accounted for, by defining the loss in density, $\psi \rho_{\alpha}^{\text {com }}$, and the corresponding flow, $\psi \rho_{\alpha}^{\text {com }} u^{e}\left(\rho_{\alpha}\right)$, at $X_{\text {Exit }}$. Following multi-lane macroscopic fluid dynamic partial differential equations (Equations 6, 7 and 8), propose an integrated model that defines this behavior and reflects the effect of communication on mobility. Equations 6 and 7, are deduced from Equations 1 and 2, respectively. Note that the Kronecker Delta ensures that the new interaction (exiting) can only occur at the Exit. $\rho^{E x i t}$ indicates the cars that were going to exit independent of congestion (because their route lies along the Exit), and $\rho_{\alpha}^{\text {comExit }}$ denotes CECs that are similarly pre-destined to exit.

Finally, we also need a new equation (8) for representing the fact that the density of radio-enabled cars that exit is withdrawn from the flow that continues beyond the Exit. This drop in the number of CECs propagates down the freeway with time. The 


$$
\begin{aligned}
& \partial_{t}\left(\rho_{\alpha}\right)+\partial_{x}\left(\rho_{\alpha} u_{\alpha}\right)=\left(\frac{1}{T_{\alpha-1}^{L}} \rho_{\alpha-1}-\frac{1}{T_{\alpha}^{R}} \rho_{\alpha}\right)\left(1-\delta_{\alpha, 1}\right)+\left(\frac{1}{T_{\alpha+1}^{R}} \rho_{\alpha+1}-\frac{1}{T_{\alpha}^{L}} \rho_{\alpha}\right)\left(1-\delta_{\alpha, N}\right) \\
& -\delta_{x, X_{E x i t}}\left[\psi \rho_{\alpha}^{c o m}+\rho_{\alpha}^{E x i t}\right] \\
& \partial_{t}\left(\rho_{\alpha} u_{\alpha}\right)+\partial_{x}\left(p^{e}\left(\rho_{\alpha}\right)+\rho_{\alpha} u_{\alpha}^{2}\right)+\partial_{x}\left(A^{e}\left(\rho_{\alpha}\right)\right)=\left(\frac{1}{T_{\alpha-1}^{L}} \rho_{\alpha-1} u_{\alpha-1}-\frac{1}{T_{\alpha}^{R}} \rho_{\alpha} u_{\alpha}\right)\left(1-\delta_{\alpha, 1}\right) \\
& +\left(\frac{1}{T_{\alpha+1}^{R}} \rho_{\alpha+1} u_{\alpha+1}-\frac{1}{T_{\alpha}^{L}} \rho_{\alpha} u_{\alpha}\right)\left(1-\delta_{\alpha, N}\right)+\frac{1}{T^{e}\left(\rho_{\alpha}\right)} \rho_{\alpha}\left[u^{e}\left(\rho_{\alpha}\right)-u_{\alpha}\right] \\
& -\delta_{x, X_{E x i t}}\left[\psi \rho_{\alpha}^{c o m} u^{e}\left(\rho_{\alpha}\right)+\rho_{\alpha}^{E x i t} u^{e}\left(\rho_{\alpha}\right)\right] \\
& \partial_{t}\left(\rho_{\alpha}^{c o m}\right)+\partial_{x}\left(\rho_{\alpha}^{c o m} u_{\alpha}\right)=\left(\frac{1}{T_{\alpha-1}^{L}} \rho_{\alpha-1}^{c o m}-\frac{1}{T_{\alpha}^{R}} \rho_{\alpha}^{c o m}\right)\left(1-\delta_{\alpha, 1}\right)+\left(\frac{1}{T_{\alpha+1}^{R}} \rho_{\alpha+1}^{c o m}-\frac{1}{T_{\alpha}^{L}} \rho_{\alpha}^{c o m}\right)\left(1-\delta_{\alpha, N}\right) \\
& -\delta_{x, X_{E x i t}}\left[\psi \rho_{\alpha}^{\text {com }}+\rho_{\alpha}^{\text {comExit }}\right]
\end{aligned}
$$

reduction in $\rho_{\alpha}^{\text {com }}$ defines the loss in number of CECs on the freeway and the subsequent reduction in network connectivity. Equation (8) is similar to (1), as $\rho_{\alpha}^{c o m}$ is a fraction of $\rho_{\alpha}$, and its nature is intuitively, similar to that of $\rho_{\alpha}$.

\section{SOlutions AND Numerical Verification}

The system of equations presented above is difficult to solve analytically, and the form of such a solution, if possible, is not likely to provide good insight into the character of the solution. Fortunately, these fluid-dynamic equations belong to a system of hyperbolic conservation laws [25], and numerical methods are able to provide approximate solutions for these, with realistic initial and boundary conditions. We solve the fluid dynamic equations, using Lax-Wendroff's 3-point finitedifference method [26] which provides a stable solution for the fluid-dynamic partial differential equations. The model equations are solved in MATLAB.

As a baseline test, we solve the model with no CECs, and with lane closure. We obtain solutions that are exactly comparable to the solutions obtained in [10], [22]. Figures 1(a) and (b) show these solutions. When the fraction $f^{c o m}$ of CECs is increased, it is expected that some cars will exit, and escape the congestion. Thus the congestion will be ameliorated. This is indeed observed when $f^{c o m}$ is high. However, for intermediate values of $f^{c o m}$, and somewhat aggressive driver behavior in responding to the congestion message, an unexpected effect is observed. Equation (8) provides the first hint that the broad character of the traffic behavior might change as a consequence of the congestion notification. Fig. 2 shows the solution to the above equations for a specific such case. In this $f^{c o m}=0.8$ and $\Phi$ is very high, so most drivers who hear the congestion message take the exit.

These figures show that the exit acts as a source of oscillation which travel down the highway. Figure 2(c) plots the phenomenon further into time, and shows that these oscillations continue and do not die down.

Tracing the evolution of the flow allows us to understand the source of the oscillations. As congestion builds up, the notification is sent back to the CECs behind the Exit. As these cars exit, the radio density in the region immediately beyond the Exit falls, which causes disconnection. No con- gestion notification is available to the CECs now behind the Exit, which now flow into the congestion, restoring connection again. Note that the oscillations are not really due to the congestion periodically being relieved, but only the information regarding the congestion being unavailable. Interestingly, we have verified that introducing a temporal persistence in the congestion notifcation can delay individual disconnections but cannot eliminate oscillation. We do not present details of this investigation for the sake of brevity.

As further validation of our model, we have also implemented a complete microscopic level simulation of the microscopic model in Java, based on a discrete event model representing individual driving decisions by individual cars. The general nature of the simulation results agree very well with our model solutions. A sample simulation result, corresponding to the case of Figure 1(a), is shown in Figure 1(c).

\section{CONCLUSION}

We have developed a model of vehicular mobility that integrates the effect of a congestion amelioration algorithm operating on a V2V network. We have shown that our model is successful in representing the various interactions between the mobility and communication of different cars. Our model is able to accommodate different fractions of CECs among the total number of cars (useful because deployment is likely to be incremental), and a large range of possible driver responses. The solutions of our model under various initial conditions are as expected and reasonable, and validated by microscopic simulation - a significant achievement in itself. We were also able to show using our model that under certain circumstances a previously unsuspected oscillation can arise, where the communication network is periodically defeated by its own success in ameliorating congestion. In future, we hope to extend this work to more general roadway topologies, and examine other intelligent roadway applications for their effect on mobility.

\section{REFERENCES}

[1] The FleetNet Project, "http://www.fleetnet.de."

[2] The PATH Project, "http://www.path.berkeley.edu."

[3] The DSRC Project, "http://www.leearmstrong.com/dsrc/dsrchomeset.htm."

[4] Vehicle Information and Communication System Center (VICS), "http://www.vics.or.jp/english/." [Online]. Available: http://www.vics.or.jp/english/ 


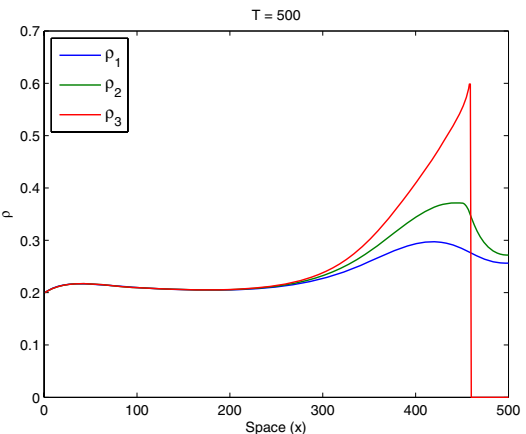

(a) Density, $t=500$

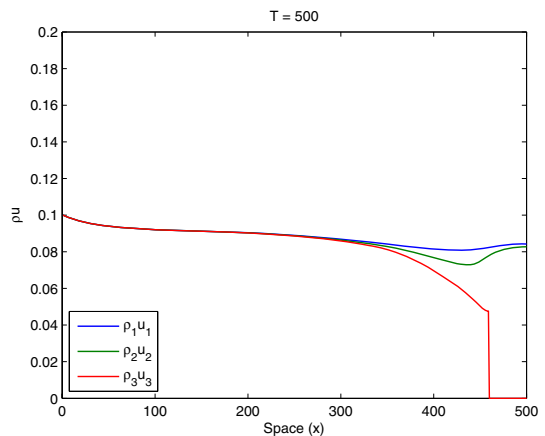

(b) Flow, $t=1000$

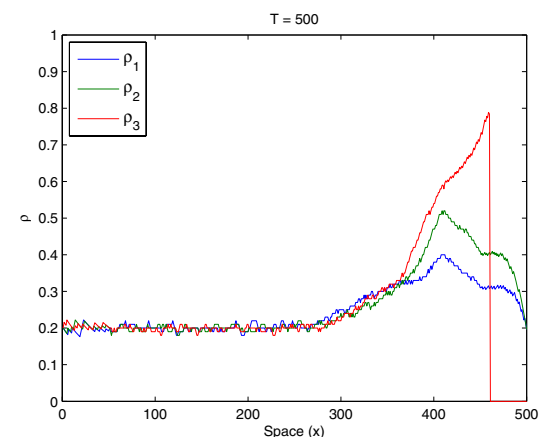

(c) Density from simulation

Fig. 1. Base Case of Traffic Flow, lane closure, no CECs

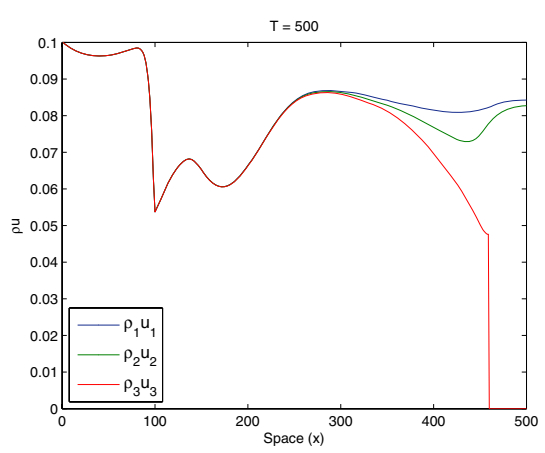

(a) $t=500$

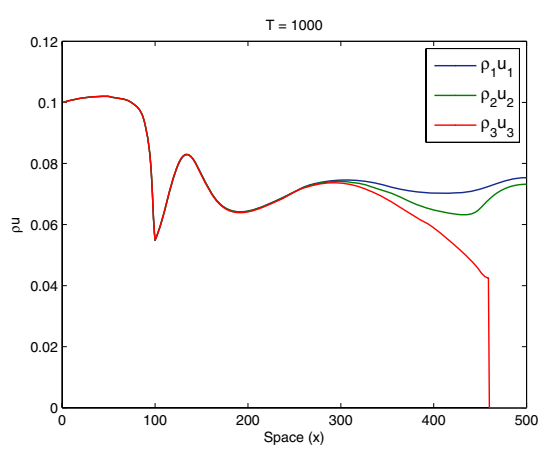

(b) $t=1000$

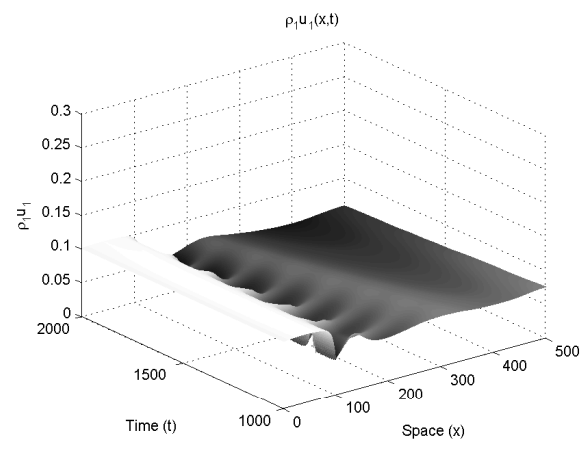

(c) $t=1000$ to 2000

Fig. 2. Oscillating behavior of traffic flow $\rho u$ under congestion notification

[5] Advanced Cruise-Assist Highway Systems (AHS), "http://www.ahsra.or.jp/." [Online]. Available: http://www.ahsra.or.jp/

[6] I. $\quad 802.11 \mathrm{p}$ Task Group, "http://grouper.ieee.org/groups/scc32/dsrc/index.html."

[7] Car2Car Communication Consortium, "http://www.car-to-car.org/."

[8] Martin Treiber, Ansgar Hennecke, and Dirk Helbing, "Congested traffic states in empirical observations and microscopic simulations," in PHYSICAL REVIEW E, vol. 62(2), August 2000.

[9] A. Kesting, M. Treiber, and D. Helbing, "Game-theoretic approach to lane-changing in microscopic traffic models," in Submitted to Transportation Research B, 2006

[10] Axel Klar and Raimund Wegener, "A Hierarchy of Models for Multilane Vehicular Traffic I: Modeling," in Society for Industrial and Applied Mathematics, vol. 59, 1999, pp. 1002-1011.

[11] I.Prigogine, "A Boltzmann-like Approach to the Statistical Theory of Traffic Flow," in Hermand,R.(ed), Theory of Traffic Flow, 1961.

[12] D. Helbing, "Gas-kinetic derivation of Navier-Stokes-like traffic equations," in Physical Review E, vol. 53, 1996, pp. 2366-2381.

[13] P. Martin, J. Perrin, B. Hansen, R.Kump, and D.Moore, "Incident Detection Algorithm Evaluation," in Report for Utah Department of Transportation, March 2001.

[14] E. Parkany andC Xie, "A Complete Review of Incident Detection Algorithms and Their Deployment: What Works and What Doesnt," in Prepared for The New England Transportation Consortium, NETCR37 Project No. 00-7, February 2005.

[15] L. Briesemeister and G. Hommel, "Localized Group Membership Service for Ad Hoc Networks," in Proceedings of the International Conference on Parallel Processing Workshops (ICPPW02),IEEE, 2002.

[16] L. Briesemeister, "Group Membership and Communication in Highly Mobile Ad Hoc Networks," in PhD thesis, School of Electrical Engineering and Computer Science, Technical University of Berlin, Germany, November 2001.

[17] H. Wu, R. Fujimoto, R. Guensler, and M. Hunter, "MDDV: a mobilitycentric data dissemination algorithm for vehicular networks," in Proc. of the first ACM workshop on Vehicular ad hoc networks, VANET'04, 2004, pp. 47-56.

[18] S.Das, A.Nandan, G.Pau, M. Sananadidi, and M. Gerla, "SPAWN: a swarming protocol for vehicular ad-hoc wireless networks," in Proc. of the first ACM workshop on Vehicular ad hoc networks, 2004, pp. 93-94.

[19] L. Briesmeister and G. Hommel, "Role-based multicast in highly mobile but sparsely connected ad hoc networks," in Proc. of the First Annual Workshop on Mobile and Ad Hoc Networking and Computing, MobiHoc'00, 2000, pp. 45-40.

[20] A. Bachir and A. Benslimane, "A Multicast Protocol in Ad Hoc Networks: Inter-Vehicle Geocast," in The 57th IEEE Semiannual Vehic. Tech. Conf., 2003.

[21] Serge P. Hoogendoorn, "State-of-the-art of Vehicular Traffic Flow Modelling," in Special Issue on Road Traffic Modelling and Control of the Journal of Systems and Control Engineering, Delft University of Technology.

[22] Axel Klar and Raimund Wegener, "A Hierarchy of Models for Multilane Vehicular Traffic II: Numerical Investigations," in Society for Industrial and Applied Mathematics, vol. 59, 1999, pp. 1002-1011.

[23] Panos G. Michalopoulos, Richard D. Jacobson, Craig A. Anderson, and Thomas B. DeBruycker, "Automatic Incident Detection Through Video Image Processing," in Traffic Engineering + Control, February 1993.

[24] David Choffnes and Fabin E. Bustamante, "STRAW - An Integrated Mobility and Traffic Model for VANETs," in Proc. of the 10th International Command and Control Research and Technology Symposium (CCRTS), vol. 62(2), June 2005. [Online]. Available: http://www.aqualab.cs.northwestern.edu/projects/STRAW/index.php

[25] Randall J. LeVque, "Systems of conservation laws," in Numerical Methods for Conservation Laws, Published by Birkhuser.

[26] P. Lax and B. Wendroff, "Systems of conservation laws," in Commun. Pure Appl. Math., vol. 13, June 1960, pp. 217-237. 